\title{
Análise fitoquímica dos metabolismos secundário da raiz e folha da Planta
}

\section{Phyllanthus Niruri L. (Quebra-pedra)}

\author{
Phytochemical Analysis of Secondary Root Metabolisms and Plant Leaf Phyllanthus Niruri L.
} (Stone Breaker)

Análisis fitoquímico del metabolismo secundario de la raíz y la hoja de la planta Phyllanthus Niruri

L. (rompepiedras)

Idonilton da Conceição Fernandes ORDCID: https://orcid.org/0000-0003-4728-4488 Universidade Federal do Paraná, Brasil E-mail: idoniltonton@gmail.com Diorges Boone da Silva ORDCID: https://orcid.org/0000-0001-7129-4534 Universidade de São Paulo, Brasil E-mail: diorgesboone@usp.br

André Luiz Freitas Nogueira

ORCID: https://orcid.org/0000-0002-4698-8566 Centro Universitário Fametro, Brasil E-mail: andreefreitas@ outlook.com Patrícia da Silva Araújo

ORDCID: https://orcid.org/0000-0001-6819-898X

Faculdades Doctum de Serra, Brasil E-mail: araujo.pate@gmail.com

Emelly Cristina Silva Rodrigues ORDCID: https://orcid.org/0000-0002-9512-7236 Singular Educacional, Brasil E-mail: emellysr@hotmail.com
Dyanna Prestes da Costa ORDCID: https://orcid.org/0000-0002-2984-9070 Centro de Formação, Pós-Graduação e Pesquisa em Saúde, Brasil E-mail: day-prestes@hotmail.com

Anderson Lima Cordeiro da Silva ORDCID: https://orcid.org/0000-0001-6777-0622 Universidade de São Paulo, Brasil E-mail: andersoncordeiro@usp.br

\begin{abstract}
Resumo
Objetivo: Realizar análise fitoquímica dos metabolismos secundários da raiz e folha da Planta Phyllanthus Niruri L. (Quebra-pedra). Métodos: Os procedimentos experimentais, bem como a análise fitoquímica seguiram as recomendações evidenciadas no manual de Barbosa et al., (2001) intitulado "Manual Para Análise Fitoquímica e Cromotográfica de Extratos Vegetais". A coleta da planta Phyllanthus Niruri vulgo "quebra-pedra" se deu a partir do mês de março de 2015 até abril do mesmo ano, no município de Boa Vista-Roraima. O processo de estudo iniciou com a secagem da planta por cinco dias em ambiente sem interferência da luz solar, posteriormente, se deram a análise dos metabolismos utilizando partes da folha e raiz, o peso bruto da folha era equivalente a $1,680 \mathrm{~g}$ e a raiz apresentava 0,103g. Resultados: Foi possível mostrar através do experimento a presença de taninos, flavonoides. Os resultados obtidos foram para folha positivo para taninos, flavonoides e para raiz positivo apenas para flavonoide. Conclusão: Os resultados ajudam a montar uma estratégia de auxiliar na procura de novos insumos que tem em vista a relação de custo benefício além de contribuir para futuras pesquisa com as plantas medicinais.
\end{abstract}

Palavras-chave: Cálculos renais; Fitoterapia; Assistência farmacêutica.

\begin{abstract}
Objective: Perform phytochemical analysis of the secondary metabolisms of the root and leaf of the plant Phyllanthus Niruri L. (Stone Breaker). Methods: The experimental procedures as well as the phytochemical analysis followed the recommendations evidenced in the manual of Barbosa et al., (2001) entitled "Manual for Phytochemical and Chromotographic Analysis of Plant Extracts". The collection of the plant Phyllanthus Niruri, known as "stonebreaker", took place from March 2015 until April of the same year, in the city of Boa Vista-Roraima. The study
\end{abstract}


process began with the drying of the plant for five days in an environment without interference from sunlight, then the analysis of metabolisms using parts of the leaf and root, the gross weight of the leaf was equivalent to $1.680 \mathrm{~g}$ and the root was $0.103 \mathrm{~g}$. Results: It was possible to show through the experiment the presence of tannins, flavonoids. The results obtained were for tannin-positive leaf, flavonoid and for flavonoid-positive root only. Conclusion: The results help to set up a strategy to help in the search for new inputs that has in view the cost-benefit ratio and contribute to future research with medicinal plants.

Keywords: Kidney calculi; Phytotherapy; Pharmaceutical services.

\section{Resumen}

Objetivo: Realizar un análisis fitoquímico de los metabolismos secundarios de la raíz y la hoja de la planta Phyllanthus Niruri L. (Stone Breaker). Métodos: Tanto los procedimientos experimentales como el análisis fitoquímico siguieron las recomendaciones evidenciadas en el manual de Barbosa y otros (2001) titulado "Manual para el análisis fitoquímico y cromográfico de los extractos de plantas". La recolección de la planta Phyllanthus Niruri, conocida como "rompehuesos", tuvo lugar desde marzo de 2015 hasta abril del mismo año, en la ciudad de Boa Vista-Roraima. El proceso de estudio comenzó con el secado de la planta durante cinco días en un ambiente sin interferencia de la luz solar, luego el análisis de los metabolismos utilizando partes de la hoja y la raíz, el peso bruto de la hoja equivalía a $1.680 \mathrm{~g}$ y la raíz a $0.103 \mathrm{~g}$. Resultados: Fue posible mostrar a través del experimento la presencia de taninos, flavonoides. Los resultados obtenidos fueron para la hoja positiva en tanino, el flavonoide y para la raíz positiva en flavonoides solamente. Conclusión: Los resultados ayudan a establecer una estrategia para ayudar en la búsqueda de nuevos insumos que tenga en cuenta la relación costo-beneficio y contribuir a la futura investigación con plantas medicinales.

Palabras clave: Cálculos renales; Fitoterapia; Servicios farmacéuticos.

\section{Introdução}

Do ponto de vista histórico, as plantas eram utilizadas de maneira alimentícia pelo homem para garantia de sobrevivência. Com o passar do tempo, passaram a ser empregadas como matéria prima para fabricação de utensílios e ferramentas, bem como na confecção de roupas. No Brasil, o manuseio das plantas como medida fitoterápica passou a ser inserida na sociedade por intermédio da comunidade indígena ainda no período da colonização para tratamento de diversas patologias, influenciada pela sociedade europeia da época, essas medidas aos poucos foram substituídas por medicamentos importados com garantia de resposta a cura rápida (Braga, 2011; Bruning et al., 2012).

Desde 1978 com a declaração de Alma-Ata que preconizava a necessidade de valorização das plantas medicinais no âmbito sanitário, publicada durante a realização da Conferência Internacional sobre Cuidados Primários em Saúde no Cazaquistão, foram normatizadas e regulamentadas políticas que abordavam a temática. Em 2006, o Brasil com objetivo de sensibilizar o uso racional da fitoterapia, bem como o acesso seguro foi criado a Política e Programa Nacional de Plantas Medicinais e Fitoterápicos por meio do Decreto № 5.813 (Mendes, 2004; Brasil, 2006; 2015).

A utilização de plantas para fins medicinais, vem despertando grande interesse, visto que sua composição química dispõe de muitos benefícios, sendo bastante estudada para fins econômicos e na busca de novos insumos medicamentosos (Silva, 2010). De tal modo, o desenvolvimento científico e tecnológico tem demonstrado interesse pelo conhecimento farmacológico das plantas medicinais, assim como investimentos para que novos princípios ativos sejam descobertos para ações terapêuticas (Oliveira et al., 2010).

De acordo com a análise fitoquímica realizada por Marques (2010) em planta do gênero Phyllanthus Niruri L. vulgo "Quebra-Pedra" foi possível observar que a planta detém de amplas espécies e apresenta excelentes resultados no tratamento em pacientes acometidos com cálculos renais, comumente utilizado na forma de chá em especial pela comunidade rural e ribeirinha por conta da dificuldade em acesso ao serviço de saúde.

Em levantamento bibliográfico realizado por Nabas et al. (2015), pode-se afirmar que a planta quebra-pedra, possui propriedades e resultados farmacológicos comprovadamente eficientes e que não provém apenas de crença popular. A planta tem ação analgésica e de relaxamento da uretra facilitando a expulsão dos cálculos sem dor e sangramento, instigando o aumento da filtração glomerular e na excreção de ácido úrico. 
Comumente, os sintomas apresentados pelos pacientes acometidos pela patologia em questão são, cólicas e desconforto urinário podendo esses estar diretamente ligado à obstrução aguda do sistema urinário, sendo assim, evidenciados com maior frequência a partir de o desprendimento dos cálculos da parede dos rins direcionando-os a bexiga. Com isso, a indicação de tratamento médico passa a ser a base de analgesia e antibioticoterapia, e em casos mais graves indica-se intervenções cirúrgicas (Sampaio; Filho, 2000).

Estudo de Almeida (2017), denota a metabolização que são reações químicas dos seres vivos que ocorrem dentro das células, sendo que, as células vegetais dividem-se em metabolismo primário desempenhando função de fotossíntese, respiração e transporte, já o metabolismo secundário exerce função de conversão de energia, possuindo finalidade de reação das plantas a diferentes tipos de ambientes. Estudos sobre o metabolismo secundário, tem se tornado crescentes nas últimas décadas, visto que há uma grande biodiversidade na terapêutica cultural para os seres humanos.

Os testes fitoquímicos, são estudos dos princípios ativos dos vegetais, também são conhecidos como metabólitos secundários ou metabolitos especiais, podendo citar como mais estudados os: alcaloides, flavonoides, taninos, antraquinonas, etc. Estas análises, fornecem conhecimentos sobre os metabolitos das plantas, de forma a isolar os princípios ativos fundamentais para produção de novos medicamentos, objetivando a extração (Bessa et al., 2013).

Elementos que constituem a biodiversidade, estão as plantas medicinais que são utilizadas em comunidades tradicionais que exercem ações terapêuticas com grandes valores para saúde e economia em pessoas que sobrevivem de recursos naturais, como remédios caseiros, medicamentos (Firmo et al., 2011).

De modo geral, as pesquisas com plantas crescem de forma significativa, salientando que a comprovação de sua eficácia contribui para estes estudos, que são conhecidos como fitoquímica, abrangendo pesquisas com vegetais, potencializando a descoberta de outros princípios ativos e substâncias. (Klein, 2014).

\section{Metodologia}

Trata-se de um estudo descritivo, analítico de cunho quali-quanti. Pereira et al. (2018), caracteriza este tipo de pesquisa como métodos importantes que requerem a interpretação do pesquisador acerca do fenômeno de estudo, bem como utiliza-se a medição de grandezas e obtém-se por meio da metrologia, números com suas respectivas unidades.

A coleta da planta Phyllanthus Niruri vulgo "quebra-pedra" se deu a partir do mês de março de 2015 até abril do mesmo ano, no município de Boa Vista-Roraima. O processo de estudo iniciou com a secagem da planta por cinco dias em ambiente sem interferência da luz solar, posteriormente, se deram a análise dos metabolismos utilizando partes da folha e raiz, o peso bruto da folha era equivalente a $1,680 \mathrm{~g}$ e a raiz apresentava $0,103 \mathrm{~g}$.

Foram aplicadas medidas de elegibilidade e inelegibilidade de acordo com a apresentação da folha, sendo consideradas inapropriadas para os estudos as que tinham características mofadas, de forma contrária, eram trituradas e pulverizadas com a adição de $30 \mathrm{~mL}$ de etanol e levadas à fervura por cerca de 5 minutos. Tal processo era sucedido pela filtração no qual foi possível obter 25,5 do extrato sendo adicionado mais 24,5 de etanol para completar $50 \mathrm{~mL}$ da extração para a análise.

A partir da iminência até a raiz foi adicionado $10 \mathrm{~mL}$ de etanol, posteriormente submetido à fervura por cerca de 5 minutos, a partir do tempo de espera foi realizado o processo de filtragem obtendo $20 \mathrm{~mL}$ extraída da raiz, adicionado mais 10 $\mathrm{mL}$ de etanol para totalizar o equivalente a $30 \mathrm{~mL}$ para a análise.

Sendo assim, a partir dos extratos obtidos foi possível dividir $0,5 \mathrm{~mL}$ para nove tubos de ensaio referente a raiz e 3,5 $\mathrm{mL}$ com extratos da folha propriamente dito, podendo assim, ser iniciado o processo de análise de metabolismo secundário. 


\section{Procedimentos Experimentais}

Os procedimentos experimentais, bem como a análise fitoquímica seguiram as recomendações evidenciadas no manual de Barbosa et al., (2001) intitulado "Manual Para Análise Fitoquímica e Cromotográfica de Extratos Vegetais".

\section{Reação Com Iodo (Polissacaridio)}

A partir dos extratos obtidos anteriormente, foram adicionados equivalente a $0,5 \mathrm{~mL}$ da solução obtida da raiz mais três gotas de iodo, prospectando atingir a coloração azulada, assim também foi feito com o extrato da folha, adicionando 3,5 $\mathrm{mL}$ da mesma e duas gotas de iodo.

\section{Reação Com Água (Saponinas)}

Em um tubo de ensaio com $0,5 \mathrm{~mL}$ da solução extraída da raiz, mais $2 \mathrm{~mL}$ de água e com agitação por cerca de cinco minutos esperou-se a formação de espumas. Posteriormente, o mesmo procedimento se deu com o extrato da folha, $3,5 \mathrm{~mL}$ da solução foram adicionados com mais $1 \mathrm{~mL}$ de água e com agitação por cerca de cinco minutos.

\section{Reação Com Solução De Acetato De Chumbo (Tanino)}

Nesta fase experimental, foram adicionados em um tubo de ensaio $0,5 \mathrm{~mL}$ da solução extraída a partir da raiz com cerca de duas gotas da solução aquosa de acetado de chumbo. Dessa forma, em um segundo tubo de ensaio foi realizado a adição de 3,5 mL da solução obtida da folha mais duas gotas da solução em discussão, em ambos os procedimentos foram realizadas observações até a precipitação de castanho avermelhado denso.

\section{Reação Com Solução De Cloreto Férrico (Tanino)}

Foram dissolvidos $0,5 \mathrm{~mL}$ de extrato da raiz em três gotas de cloreto férrico, a fim de alcançar a coloração vermelha polifenol, azul ou verde. $\mathrm{O}$ mesmo procedimento foi aplicado a $3,5 \mathrm{~mL}$ do extrato obtido da folha com mais duas gotas da substancia em questão, observando-se as alterações de coloração.

\section{Reação De Shinoda Com Magnésio (Mg) (Flavonoide)}

Dissolveu-se 0,5 $\mathrm{mL}$ do extrato da raiz em fragmentos de magnésio metálico com $2 \mathrm{~mL}$ de hidróxido de amônia (pelas paredes), observou-se a evolução da coloração rósea-avermelhado indicando flavonóis e/ou violeta evidenciando a presença de flavonas. Em um segundo tubo de ensaio, foram adicionados 3,5 $\mathrm{mL}$ da solução extraída da folha, fragmentos de magnésio metálico e $2 \mathrm{~mL}$ de hidróxido de amônia (pelas paredes), esperou-se a mudança de cor sendo para rósea-avermelhados e/ou violeta.

\section{Reação Com Cloreto De Aluminio (Flavonoide)}

Cerca de $0,5 \mathrm{~mL}$ da solução extraída da raiz foram adicionadas em um tubo de ensaio com três gotas de cloreto de alumínio, em um segundo tubo de ensaio foram adicionados $3,5 \mathrm{~mL}$ mais três gotas da mesma solução aqui em discurso, as duas amostras foram submetidas a radiação luminosa ultravioleta, obtendo como resultado em ambas as amostras aspectos fluorescentes de coloração esverdeada indicando a presença de flavonoides.

\section{Reação Com Água De Cal (Antraquinona)}

Dissolveu em um tubo de ensaio $0,5 \mathrm{~mL}$ da solução extraída da raiz em $2 \mathrm{~mL}$ de água de Cal, assim foi feito também com o extrato da folha $3,5 \mathrm{~mL}$ com a adição de $2 \mathrm{~mL}$ do mesmo reagente em ambos os tubos de ensaio foram observados até a 
modificação de coloração laranja ou vermelho sangue.

\section{Reação Com Hidróxido De Amônio, Peróxido De Hidrogênio (Água Oxidenada) (Antraquinona)}

Cerca de 0,5 mL da solução extraída da raiz foram adicionadas em tubo de ensaio com mais uma gota de hidróxido de amônia e uma gota de peróxido de hidrogênio, em um segundo tubo de ensaio foram adicionados $3,5 \mathrm{~mL}$ do extrato obtido a partir da folha com uma gota de hidróxido de amônia e uma gota de peroxido de hidrogênio. Sendo assim, observou-se a modificação da coloração até a equivalência da cor vermelha.

\section{Reação Com Gelatina (Tanino)}

Separados dois tubos de ensaio, no primeiro foram adicionados $0,5 \mathrm{~mL}$ da solução extraída da raiz mais $2 \mathrm{~mL}$ de gelatina, no segundo tubo de ensaio foram diluídos $3,5 \mathrm{~mL}$ do extrato da folha em $2 \mathrm{~mL}$ de gelatina. Com isso, observou-se a precipitação.

\section{Resultados e Discussão}

A utilização da fitoterapia como método farmacológico, tem demonstrado menos efeitos adversos no que diz respeito aos medicamentos alopáticos disponíveis no mercado farmacêutico. $\mathrm{O}$ uso das plantas por parte da população é culturalmente aceita e repassada por gerações, sendo utilizada a milhares de anos antes mesmo da descoberta da substância, levando em consideração o custo benefício (Silva, 2015).

O Quadro 1, demonstra resultados advindos das reações analisadas dos flavonoides, antraquinonas, saponinas e taninos, destas apenas as saponinas não obtiveram resultados positivos. Assim, os flavonoides representam um dos grupos mais importante dos vegetais e são geralmente encontrados nas folhas, flores, raízes e frutos das plantas, sendo de suma importância para a proteção contra insetos, fungos entre outras. Os flavonoides desempenham várias atividades benéficas no ser humano, uma delas são as ações anti-inflamatórias, alguns compostos fenólicos possuem efeito inibitório das vias de ciclooxigenase (COX) e lipoxigenase, que desempenham um papel importante como mediadores anti-inflamatórios (Santos; Rodrigues, 2017).

Quadro 1. Resultados das reações na presença de metabolismos secundários para Phyllanthus niruri L.

\begin{tabular}{|l|c|c|}
\hline \multicolumn{1}{|c|}{ METABOLISMOS SECUNDÁRIOS E REAGENTES } & RAIZ & FOLHA \\
\hline Polissacarídeos Reagentes Lugol (Iodo) & NEGATIVO & NEGATIVO \\
\hline $\begin{array}{l}\text { Saponina } \\
\text { Reagente Água }\end{array}$ & NEGATIVO & NEGATIVO \\
\hline $\begin{array}{l}\text { Taninos } \\
\text { Reagente Acetado de chumbo }\end{array}$ & NEGATIVO & POSITIVO \\
\hline $\begin{array}{l}\text { Taninos } \\
\text { Reagente Cloreto Férrico }\end{array}$ & NEGATIVO & NEGATIVO \\
\hline $\begin{array}{l}\text { Flavonoides } \\
\text { Reagente reação de shinoda }\end{array}$ & POSITIVO & POSITIVO \\
\hline $\begin{array}{l}\text { Flavonoides } \\
\text { Reagente Cloreto de alumínio }\end{array}$ & NEGATIVO & NEGATIVO \\
\hline $\begin{array}{l}\text { Antraquinonas } \\
\text { Reagente água de cal, }\end{array}$ & NEGATIVO & NEGATIVO \\
\hline $\begin{array}{l}\text { Antraquinonas } \\
\text { Reagente Amônia de peroxido de hidrogênio }\end{array}$ & NEGATIVO & POSITIVO \\
\hline Polifenóis gelatina (taninos)
\end{tabular}

Fonte: Fernandes et al. (2021). 
Em um estudo realizado na espécie Phyllanthus niruri L. por Rosario e Almeida (2016), pode-se evidenciar a presença dos alcaloides, podendo julgar que o mesmo a depender do ambiente em que a espécie foi coletada pode ou não estar presente no resultado final. As mudanças climáticas, bem como o ambiente podem inferir em resultados diferentes, para mais ou para menos, visto que as plantas podem se adaptar as condições impostas, em pesquisas deste gênero é importante que as coletas sejam realizadas em um mesmo ambiente para que isso não influencie na eficácia do produto.

Dentre os produtos vegetais, o fenólico é o mais diversificado, essa classe metabólica possui um núcleo característico C6-C3- C6, por meio da estrutura básica, pode ser distribuída em diferentes subclasses como: chalconas, flavanonas, flavonas, flavonóis, isoflavonas, flavan-3-ols e antocianidinas (Coutinho et al., 2009). Os taninos são componentes distribuídos em plantas, podendo ser encontrados em alimentos e bebidas, em plantas podem estar dispostos em sementes, cascas e caules de frutos verdes, sendo muito utilizadas na indústria de alimentos como antioxidantes nos sucos. Desta forma, seus benefícios farmacológicos podem tratar muitas doenças, como problemas renais, queimaduras, processos inflamatórios e urinários (Pansera et al., 2003).

Segundo Cunha et al. (2009), Polissacarídeos são polímeros naturais, os quais podem ser constituídos de um único ou de diferentes tipos de monossacarídeos, celulose, alginato e goma arábica, podendo derivar de extração industriais de plantas, incluindo as algas, animais e fungos ou são obtidos via fermentação microbiológica. Já em plantas superiores, estes podem ser obtidos de exsudatos, sementes, frutos e tubérculos. Os polissacarídeos possuem uma ampla gama de aplicações, especialmente nas áreas de alimentos, biomédicas, farmacêutica e de cosméticos.

Entre as plantas superiores, podemos citar as saponinas, que em contato com a água a partir da agitação, geram um aspecto espumoso, normalmente os medicamentos que possuem essa substância são irritantes para as mucosas, porém são essenciais para o crescimento das plantas. Os benefícios para o ser humano estão em suas atividades anti-helmínticas, espermicidas e antifúngicas (Robbers et al., 1997).

As antraquinonas, podem apresentar-se como compostos coloridos com propriedades laxantes ou purgantes, quando utilizadas em doses altas provocam irritação intestinal, dores intensas e hipotensão, possuem anel aromáticos com duas carbonilas, usualmente uma oposta à outra, encontradas frequentemente nas famílias Rubiaceae nos vegetais, possuem ação antifúngica contra agressores e parasitas (Cunha, 2013).

De tal modo, os metabólitos secundários depsídeos e depsidonas em Phyllanthus niruri L. são biossintetizados, onde seu mecanismo envolve reação de desidratação somente na etapa final na ciclização da cadeia para a sua formação, sendo reportadas a respeito de atividades anti-inflamatória e antibiótica (Rosario; Almeida, 2016).

Um estudo experimental, in vivo, realizado em ratos com cálculos vesicais por oxalato de cálcio, por meio da utilização do chá da planta Phyllanthus niruri L. por 42 dias, demonstrou elevação do volume urinário e de creatinina no sódio urinário, além de uma redução significativa no crescimento do cálculo vesical (Santos, 1990).

Desta forma, pode-se inferir que apesar de muitas literaturas demonstrarem-se eficazes e trazer resultados relevantes no uso terapêutico em pacientes acometidos por litíase renal, processos inflamatórios e para analgesias, mostrando-se ainda com menores reações adversas se tornando de fácil acesso, por tanto sugere-se maiores complementações no que diz respeito a pesquisas com seres humanos e a disseminação do conhecimento fitoterápico. Haja visto que a pesquisa em questão, assim como outras analisadas, apresenta resultados positivos como mediadores anti-inflamatórios, o que demonstra uma eficácia quanto ao uso de plantas de forma mais natural possível para tratar doenças do aparelho geniturinário.

\section{Considerações Finais}

A utilização de plantas para fins medicamentosos, vem tornando-se cada vez mais tradicional na sociedade atual, sendo assim, a Phyllanthus ninuri L. popularmente conhecida como "Quebra-Pedra" apresenta excelentes resultados no 
tratamento de cálculos renais, com sua ação analgésica e de relaxamento da uretra facilitando a expulsão dos cálculos sem dor e sangramento, além de ser um método terapêutico de fácil acesso e de custo baixo. Após a realização dos testes fitoquímicos, foi possível a identificação dos metabólicos secundários presente na planta como polissacarídeos, saponinas, tanino, flavonoide e antraquinona que possuem ações de combater dores renais, inflamações e analgesia. $\mathrm{O}$ estudo ainda contribui apresentando resultados a partir da presença das substâncias taninos e flavonoides, além de fomentar e incentivar novos estudos acerca da temática, proporcionando maior embasamento para a comunidade cientifica ao uso seguro dessas substâncias fitoterápicas.

Frente ao exposto, espera-se contribuir de forma significativa para o meio científico com ênfase na assistência farmacêutica com base na fitoterapia, bem como busca-se servir como incentivo para a elaboração e execução de novos estudos na área, haja visto a importância da construção de mais conhecimentos acerca da temática abordada e reformulação de políticas públicas de saúde.

\section{Referências}

Almeida, D. F. L. S. (2017). Estudo das Vias Metabólicas das Plantas na Síntese de Pigmentos Naturais. Porto. Dissertação. (Mestrado em Ciências Farmacêuticas). Faculdade de Ciências da Saúde. Universidade Fernando Pessoa. Portugal.

Bessa, N. G. F., et al. (2013). Prospecção fitoquímica preliminar de plantas nativas do cerrado de uso popular medicinal pela comunidade rural do assentamento vale verde - Tocantins. Rev. Bras. Pl. Med. 15(4), 692-707.

Braga, C. M. (2011). Histórico da utilização de plantas medicinais. Brasilia. Monografia. (Graduação em Biologia licenciatura). Universidade de Brasília. Distrito Federal.

Bruning, M. C. R., et al. (2012). A utilização da fitoterapia e de plantas medicinais em unidades básicas de saúde nos municípios de Cascavel e Foz do Iguaçu - Paraná: a visão dos profissionais de saúde. Ciênc. saúde coletiva, Rio de Janeiro, 17(10), 2675-2685.

Coutinho, M. A. S., et al. (2009). Flavonoides: Potenciais Agentes Terapêuticos para o Processo Inflamatório. Rev. Virtual. Quim. 1(3) .241-256.

Cunha, L. C. S. (2013). Estudo fitoquímica, atividades antioxidante, antimicrobiana e citotóxica da espécie cassia bakeriana craibr. MG. Dissertação (Doutorado em química). Universidade Federal de Uberlândia. Uberlândia - Minas Gerais.

Cunha, P. L. R., et al. (2009). Polissacarídeos da biodiversidade brasileira: uma oportunidade de transformar conhecimento em valor econômico. Quim. Nova, 32(3), 649-660.

Klein, T. (2014). Analisefotoquímico.https://www.portaleducacao.com.br/conteudo/ artigos/farmacia/analise-fitoquimica/56896\&gt.

Marques, L. C. (2010). Phyllanthus niruri (Quebra-Pedra) no Tratamento de Urolitíase: Proposta de Documentação para Registro Simplificado como fitoterápico. Programa de Mestrado Profissional em Farmácia. Revista Fitos pag. 20 5(03).

Mendes, I. A. C. (2004). Desenvolvimento e saúde: uma declaração de Alma-Ata e movimentos posteriores. Rev. Latino-Am. Enfermagem, Ribeirão Preto, 12(3), 447-448

Ministério da Saúde. Secretaria de Ciência, Tecnologia e Insumos Estratégicos. (2006). Política nacional de plantas medicinais e fitoterápicos: Ministério da Saúde.

Ministério da Saúde. Secretaria de Atenção à Saúde. (2015). Política nacional de práticas integrativas e complementares no SUS: atitude de ampliação de acesso: Ministério da Saúde.

Nabas, J. M. A. B. B., et al. (2015). Quebra-Pedra (Phyllanthus niruri L): Considerações no Tratamento da Litíase Renal. Rev. Conexão Eletrônica - Três Lagoas, MS. 12(1).

Oliveira, C., et al. (2010). Análise fotoquímica preliminar do extrato etanoico obtido a partir do rizoma da typha domingensis pers. Revista Ceciliana Dez 2(2): 17-19.

Pansera, M. R., et al. (2003). Análises de taninos totais em plantas aromáticas e medicinais cultivadas no nordeste do Rio Grande do Sul. Rev. Bras. Farmacogn. 13(1).

Pereira, A. S., et al. (2018). Metodologia da pesquisa científica. UFSM. https://repositorio.ufsm.br/bitstream/handle/1/15824/Lic_Computacao_MetodologiaPesquisa-Cientifica.pdf?sequence $=1$.

Robbers, J. E., et al. (1997). Farmacognosia e farmacobiotecnologia. Premier. p. 372.

Rosário, A. C. A., \& Almeida, S. S. M. S. (2016). Análise fitoquímica da espécie Phyllanthus niruri L. (quebra-pedra). Estação Científica (UNIFAP).

Sampaio, F. J. B. S., \& Filho, G. D. B. Litíase Renal. Guia Prático De Urologia, Cap. 18- Litíase renal.pag 97 13/06/00.

Santos, D. R. (1990). Chá de quebra-pedra (Phyllantus niruri) na litíase urinária em humanos e em ratos. Tese de doutorado, Escola Paulista de Medicina, São Paulo. 
Research, Society and Development, v. 10, n. 4, e18410414051, 2021

(CC BY 4.0) | ISSN 2525-3409 | DOI: http://dx.doi.org/10.33448/rsd-v10i4.14051

Santos, D. S., \& Rodrigues, M. M. F. (2017). Atividades farmacológicas dos flavonoides: um estudo de revisão. Estação Científica (UNIFAP), Macapá, 7(3), 29-35.

Silva, N. L. A., et al. (2010). Triagem Fotoquímica de Plantas de Cerrado, da Área de Proteção Ambiental Municipal do Inhamum, Caxias, Maranhão. Scientia plena $6(2)$. 